\title{
Article \\ Genome-Wide Analysis of MYB10 Transcription Factor in Fragaria and Identification of QTLs Associated with Fruit Color in Octoploid Strawberry
}

\author{
Abinaya Manivannan ${ }^{+}{ }^{D}$, Koeun Han ${ }^{\dagger}$, Sun Yi Lee, Hye-Eun Lee, Jong Pil Hong, Jinhee Kim, Ye-Rin Lee, \\ Eun Su Lee (D) and Do-Sun Kim* \\ Vegetable Research Division, National Institute of Horticultural and Herbal Science, Rural Development \\ Administration, Wanju 55365, Korea; abinayamanivannan@gmail.com (A.M.); hke1221@korea.kr (K.H.); \\ sylee2@korea.kr (S.Y.L.); helee72@korea.kr (H.-E.L.); hjp3467@korea.kr (J.P.H.); sayzinni@korea.kr (J.K.); \\ lyr1219@korea.kr (Y.-R.L.); lus4434@korea.kr (E.S.L.) \\ * Correspondence: greenever@korea.kr; Tel.: +82-63-238-6670 \\ + These authors contributed equally to this work.
}

Citation: Manivannan, A.; Han, K.; Lee, S.Y.; Lee, H.-E.; Hong, J.P.; Kim J.; Lee, Y.-R.; Lee, E.S.; Kim, D.-S. Genome-Wide Analysis of MYB10 Transcription Factor in Fragaria and Identification of QTLs Associated with Fruit Color in Octoploid Strawberry. Int. J. Mol. Sci. 2021, 22, 12587.

https://doi.org/10.3390/ijms222212587

Academic Editor: Endang

Septiningsih

Received: 19 October 2021

Accepted: 19 November 2021

Published: 22 November 2021

Publisher's Note: MDPI stays neutral with regard to jurisdictional claims in published maps and institutional affiliations.

Copyright: (c) 2021 by the authors. Licensee MDPI, Basel, Switzerland. This article is an open access article distributed under the terms and conditions of the Creative Commons Attribution (CC BY) license (https:// creativecommons.org/licenses/by/ $4.0 /)$
Abstract: The genus Fragaria encompass fruits with diverse colors influenced by the distribution and accumulation of anthocyanin. Particularly, the fruit colors of strawberries with different ploidy levels are determined by expression and natural variations in the vital structural and regulatory genes involved in the anthocyanin pathway. Among the regulatory genes, MYB10 transcription factor is crucial for the expression of structural genes in the anthocyanin pathway. In the present study, we performed a genome wide investigation of MYB10 in the diploid and octoploid Fragaria species. Further, we identified seven quantitative trait loci (QTLs) associated with fruit color in octoploid strawberry. In addition, we predicted 20 candidate genes primarily influencing the fruit color based on the QTL results and transcriptome analysis of fruit skin and flesh tissues of light pink, red, and dark red strawberries. Moreover, the computational and transcriptome analysis of MYB10 in octoploid strawberry suggests that the difference in fruit colors could be predominantly influenced by the expression of MYB10 from the F. iinumae subgenome. The outcomes of the present endeavor will provide a platform for the understanding and tailoring of anthocyanin pathway in strawberry for the production of fruits with aesthetic colors.

Keywords: Fragaria; MYB10; fruit color; quantitative trait locus (QTL)

\section{Introduction}

The gene expression at transcription level is crucial for numerous physiological and biochemical processes in the biological organism. The regulation of gene transcription is determined by various factors, among which, transcription factors are predominantly important. Plant transcription factors influence the regulation of target genes that are involved in growth, development, and response to environmental stresses in plants [1]. In general, transcription factors are proteins distinguished into several families based on the structure of the DNA binding domains [2]. Transcription factors function by binding to the sequence specific cis-acting promoters to enhance or suppress the regulation of target genes. MYB is one of the important transcription factors involved in the regulation of various vital genes involved in diverse processes particularly in anthocyanin biosynthesis in plants.

In horticultural crops, biosynthesis of anthocyanin and its accumulation in tissues like flowers and fruits is important for breeding new varieties. In strawberry, the ripeness and esthetic quality of fruits are predominantly determined by the accumulation of anthocyanin [3]. Apart from the esthetic feature, anthocyanin pigments render protection against various biotic and abiotic stresses in plants [4]. The red, purple, violet, and pink pigments in fruits and flowers are due to the compounds formed from the phenylpropanoid 
pathway such as hydroxycinnamic acid, isoflavones, flavonols, phlobaphenes, and proanthocyanidins [5]. The phenylpropanoid pathway consists of several vital enzymes that are encoded by the early and late anthocyanin biosynthesis group genes such as phenylalanine ammonia lyase (PAL), chalcone hydrolase isomerase (CHI), and dihydro flavanol reductase $(D F R)$ [6]. These structural genes are influenced by the regulatory genes mainly by MYB transcription factors directly or by co-regulation with other transcription factors like bHLH and WD40 repeats by forming a MBW complex [7].

In recent years, there has been an increasing progress in the understanding of molecular mechanisms of pigmentation in fruit and flowers through multiple investigations of transcriptional regulation of key genes such as MYB transcription factors [8]. The MYB transcription factor belongs to R2R3 MYB transcription factors which play vital regulatory roles in determining the accumulation of anthocyanin in several horticultural crops including strawberry [9]. MYB transcription factors can be classified into three subfamilies based on the number of highly conserved imperfect repeats in the DNA-binding domain including R3 MYB (MYB1R) with one repeat, R2R3 MYB with two repeats, and R1R2R3 MYB (MYB3R) with three repeats [8]. Among these MYB transcription factors, R2R3-MYBs constitute the largest transcription factor gene family in plants, with more than 126 R2R3 MYB genes identified in Arabidopsis [8]. In strawberry, FaMYB10 expression caused the synthesis of high levels of anthocyanin [9]. Even though studies have identified the importance of MYB10, the genome level understanding of the MYB10 in Fragaria is still under investigation. A recent report by Castillejo et al. [10] demonstrated the allelic variation in MYB10 acts as an important factor that influences the fruit color in strawberry. The determination of genomic loci or genes controlling the variation in fruit color in octoploid strawberry is important for the breeding of new strawberry cultivars with desired esthetic fruit colors. However, the complex genome and ploidy level in cultivated strawberry pose difficulty for the investigation of genomic loci or mapping of quantitative trait loci associated with fruit colors. The recent advancements in next generation sequencing enabled the availability of the whole genome sequence of diploid and octoploid strawberry. The reference genomes of heterozygous 'Camarosa' [11] and homozygous 'Wongyo 3115' [12] cultivated strawberries can facilitate the understating of gene regulation and development of markers associated with fruit colors in octoploid strawberry.

Multiple studies were conducted to figure out the locus controlling fruit colors evaluated by visual evaluation score, color parameters $(\mathrm{L}, \mathrm{a}, \mathrm{b})$, and anthocyanin contents [10,13-17]. Total anthocyanin contents of strawberry fruits were correlated with values measured by colorimeter or the metabolites including pelagonidin derivatives. In addition, QTLs were detected to control color-related traits commonly in multiple chromosomal regions. By comparison with the physical map of $F$. vesca and F. $\times$ ananassa, MYB10, and flavonoid $3^{\prime}$ hydroxylase $\left(F 3^{\prime} H\right)$ were suggested as candidate genes for fruit color $[10,16] . F 3^{\prime} H$, a structural gene involved in flavonoid biosynthesis, showed significant correlation with flavonoid levels in $F_{1}$ lines [16]. Although, multiple QTLs were identified, possible candidate genes could be identified from genome-based QTL analysis.

The molecular insight on MYB10 can be of vital knowledge for tailoring the anthocyanin biosynthesis in strawberry. Therefore, in the present study, we have identified the MYB10 genes present in five diploids and two octoploid strawberry genomes. The transcriptome analysis of fruits from four different strawberry cultivars with different fruit colors ranging from light pink to dark red were utilized for the expression analysis of structural and regulatory genes involved in anthocyanin biosynthesis. In addition, the six QTLs associated with fruit color were detected and utilized for the prediction of candidate genes controlling the skin and flesh colors in strawberry. Overall, the outcomes of the present study can benefit the breeding of strawberry cultivars with varying fruit colors and also facilitate the development of trait specific markers for strawberry breeding. 


\section{Results}

\subsection{Characterization of MYB10 in Diploid and Octoploid Species of Fragaria}

A total of 14 MYB10 genes were identified in eight genomes of Fragaria (Table 1). All the identified MYB10 genes were located on the chromosome 1. The diploid genomes consisted of one MYB10 gene, whereas the octoploids consisted of four. The octoploid cultivated strawberry 'Wongyo 3115' consisted of four homeologs of MYB10 located in each subgenome, whereas 'Camarosa' consisted one MYB10 homeolog in each Ch1-1, Ch1-2 and Ch1-3 encompassed two homeologs of MYB10, whereas no MYB10 was identified in Ch1-4. In homozygous octoploid strawberry, two MYB10 (g00115370 and g00119641) were annotated as MYB113 in the 'Wongyo 3115' annotation file. The gene size of MYB10 ranged from 1.8 to $3.2 \mathrm{~kb}$ in diploids and 0.4 to $11.1 \mathrm{~kb}$ in octoploid strawberry. Among the diploids, F. nilgerensis consisted of the largest MYB10 with $3.2 \mathrm{~kb}$ and F. iinumae encompassed the smallest MYB10 with gene size of $1.8 \mathrm{~kb}$. In homozygous octoploid strawberry 'Wongyo 3115', the smallest MYB10 homeolog g00101712 comprised of $467 \mathrm{bp}$, whereas the other MYB10 homeologs were of similar size to the diploids. Similarly, in heterozygous octoploid strawberry 'Camarosa' the MYB10 homeolog Fvb1-3-augustus-gene-143.29 (143.29) consisted of $331 \mathrm{bp}$ whereas the size of Fvb1-2-snap-gene-157.15 (157.15) MYB10 homeolog consisted of $11.1 \mathrm{~kb}$.

Table 1. Characterization of MYB10 transcription factor in Fragaria.

\begin{tabular}{lcccc}
\hline Species & Chr. & Gene ID & Location & Ploidy Level \\
\hline F. iinumae & 1 & evm.model.scaf_28.444 & $14,574,366-14,576,255$ & Diploid \\
\hline F. nilgerensis & 1 & evm.model.ctg92.252_scbg_v1.0 & $17,100,850-17,104,063$ & Diploid \\
\hline F. nubicola & 1 & evm.model.ctg173.372 & $15,072,390-15,075,138$ & Diploid \\
\hline F. viridis & 1 & evm.model.ctg108.134 & $13,877,866-13,880,523$ & Diploid \\
\hline F. vesca & 1 & FvH4_1g22020 & $13,950,290-13,952,507$ & Diploid \\
\hline & $1-1$ & $g 00101712$ & $13,223,169-13,223,636$ \\
\cline { 2 - 5 } F. $\times$ ananassa 'Wongyo 3115' & $1-2$ & g00119641 & $12,622,806-12,626,007$ \\
\cline { 2 - 5 } & $1-3$ & g00115370 & $13,537,353-13,539,011$ \\
\hline & $1-4$ & Octoploid & $13,235,156-13,238,279$ \\
F. $\times$ ananassa 'Camarosa' & $1-1$ & maker-Fvb1-1-snap-gene-139.18 & $13,899,904-13,911,034$ \\
\cline { 2 - 5 }
\end{tabular}

\subsection{Structure and Phylogeny of MYB10 in Diploid and Octoploid Species of Fragaria}

After the identification of MYB10 in diploid and octoploid Fragaria species, the gene structure and motifs were analyzed (Figure 1). Gene structure of MYB10 consisted of three exons separated by two introns. All the diploids consisted of three exons and two introns. However, in homozygous octoploid F. $\times$ ananassa 'Wongyo 3115' two MYB10 ( $g 00115370$ and g00119641) homeologs encompassed similar exons and introns like diploids. The g00101712 displayed only one exon and g00128511 possessed one intron and two exons. In the heterozygous octoploid strawberry $F . \times$ ananassa 'Camarosa' the two homeologs makerFvb1-3-augustus-gene-144.30 (144.30) and 157.15 displayed similarity with the diploids, whereas two exons and one snap_masked-Fvb1-3-processed-gene-138.18 (139.18) intron were identified in $F . \times$ ananassa 'Camarosa' 143.29. On the other hand, six exons spaced by five introns were identified in the homeolog 139.18. Further, motif analysis using the MEME tool demonstrated the occurrence of three conserved motifs in most of the MYB10 proteins of Fragaria except in F. nipponica, F. $\times$ ananassa 'Wongyo 3115' (g00128511 and g00101712), and $F . \times$ ananassa 'Camarosa' (143.29). 

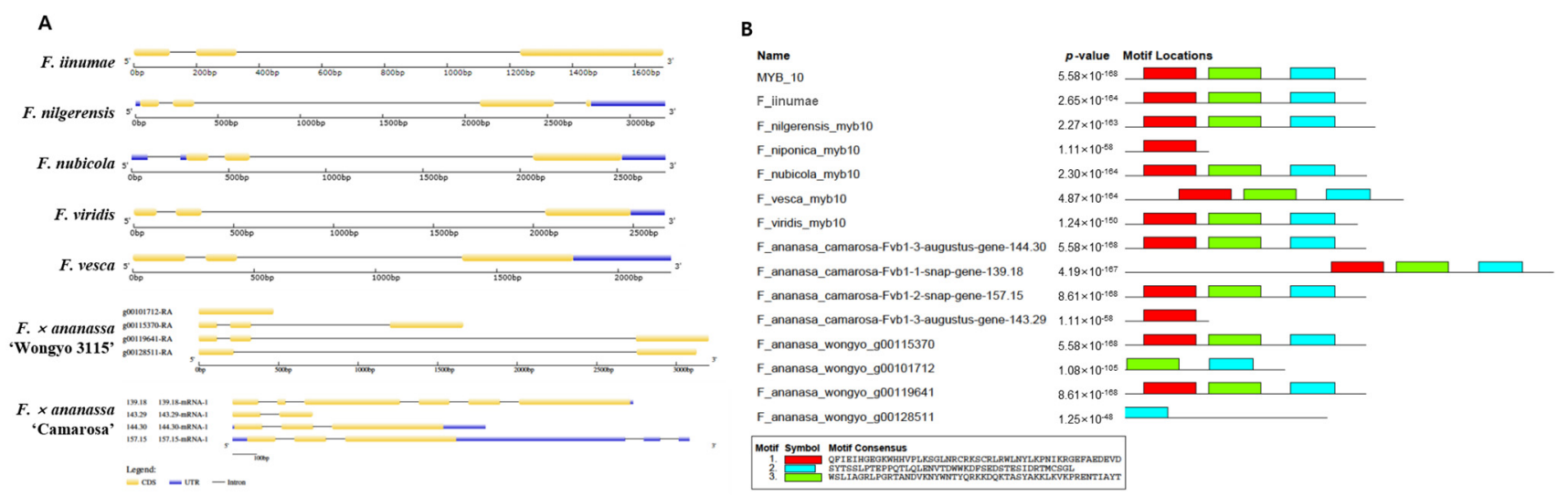

Figure 1. Structure of MYB10 in diploid and octoploid Fragaria (A) and motifs identified in Fragaria MYB10 (B).

The multiple sequence alignment of MYB10 proteins suggested the presence of signature R2 and R3 domains in Fragaria (Figure 2A). However, the R2 domain and partial $\mathrm{R} 3$ domains were absent in $F . \times$ ananassa 'Wongyo 3115' (g00101712). In addition, two conserved motifs A [ANDV] and B [RPRPRTF] required for the activity of MYB10 was identified. According to previous report, the presence of motif $A$ is vital for the interaction with bHLH co-factors for the promotion of anthocyanin biosynthesis [18], whereas the C-terminal conserved motif $B$ is important for regulation of anthocyanin genes [18]. The conserved motif A and B were absent in F. nubicola, F. $\times$ ananassa 'Camarosa' (143.29), and F. $\times$ ananassa 'Wongyo 3115' (g00128511) MYB10 transcription factors. The first arginine residue was substituted by lysine in motif B of $F . \times$ ananassa 'Wongyo 3115' (g00101712) and the third arginine residue was replaced by glutamine in the g00115370. Further, to investigate the phylogenetic relationship among Fragaria species, a dendrogram was constructed for the amino acid sequence of MYB10 using maximum likelihood algorithm (Figure 2B). The tree displayed two major clusters with most of the diploids such as F. iinumae, F. vesca, F. viridis, F. nilgerensis, and F. nubicola along with the octoploid F. $\times$ ananassa 'Camarosa' (144.30 and 157.15) and F. $\times$ ananassa 'Wongyo 3115' (g00115370 and g00119641) grouped in cluster 1. Furthermore, cluster 2 consisted of diploid F. nipponica, octoploids $F$. $\times$ ananassa 'Camarosa' (143.29 and 139.18), and F. $\times$ ananassa 'Wongyo 3115' (g00128511 and g00101712). The clusters 1 and 2 are further divided into five and four sub-groups, respectively. MYB10 from F. iinumae displayed a close relationship with $F . \times$ ananassa 'Wongyo 3115' (g00119641) and F. $\times$ ananassa 'Camarosa' (157.15). Other diploids, such as F. vesca, F. viridis, F. nilgerensis, and F. nubicola, exhibited a close evolutionary relationship.

\subsection{RNA-Seq Based Expression Profiling of MYB10 and Anthocyanin Biosynthesis Genes}

The differential expression profile of 28 important genes including both structural and regulatory genes involved in anthocyanin biosynthesis in the skin and flesh tissues of three strawberry cultivars were investigated (Figure 3, Table S1). The three cultivar 'Mannyeonsul', 'Maehyang', and 'P69' had light pink, red, and dark red fruit color and showed difference in $\mathrm{L}^{*}$ (brightness) and $\mathrm{a}^{*}$ (redness) value (Figure 3, Table S2). The MYB10 homeolog from Ch1-2 (g00119641) contributed by F. iinumae subgenome of octoploid strawberry displayed higher relative expression levels, whereas mere expression of Ch1-3 (g00115370) was observed in the transcriptome analysis. However, no significant expression of the other MYB10 genes were identified. The transcriptome results suggested the higher expression of most of the structural genes, such as PAL (g00141093), glycosyltransferase (GT; g00138590), flavonoid glucosyltransferase (FGT; g00134528), 4-coumaroyl-CoA ligase-like (4CLL; g00016283), CHI3 (g00150431), CHI2 (g00133127), and chalcone synthase (CHS; g00138715), were observed in the skin and flesh tissues of dark red 'P69'. On the other hand, the light pink 'Mannyeonsul' displayed a lower expression of the vital structural genes involved in anthocyanin biosynthesis. The higher transcript levels of DFR ( $g 00076873, g 00055948$, and g00093085) and anthocyanidin synthase (ANS; g00056454) were identified in the skin tissue 
of red fruit bearing 'Maehyang' cultivar. Further, increase in the abundance of the MYB10 transcript (g00119641) was observed in skin and flesh tissues of 'P69' while MYB10 displayed lower expression in the light pink fruits of the 'Mannyeonsul' cultivar. The expression of $b H L H$ transcription factor bHLH149 (g00015055 and g00002221) and bHLH146 (g00122119) were higher in both the tissues of 'P69' and lower in 'Mannyeonsul'. However, the bHLH78 (g00053443) expression was least in skin and flesh tissues of 'Maehyang' cultivar.

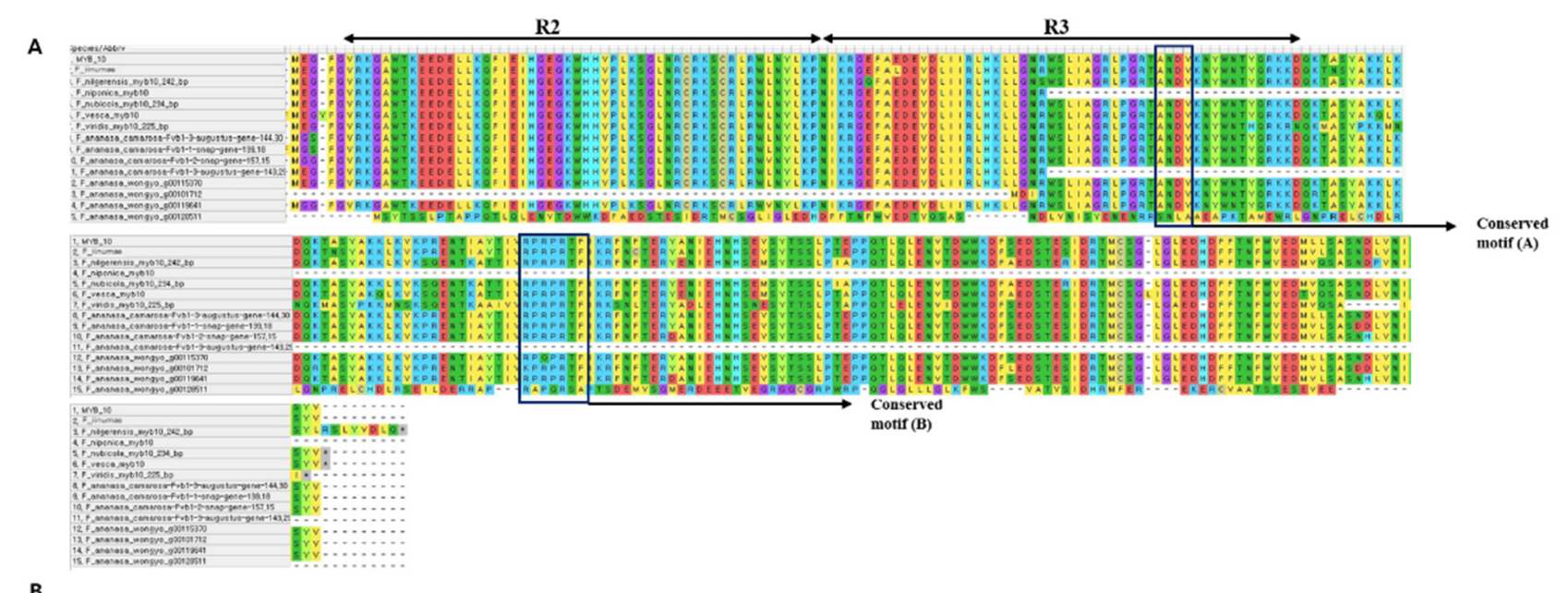

B

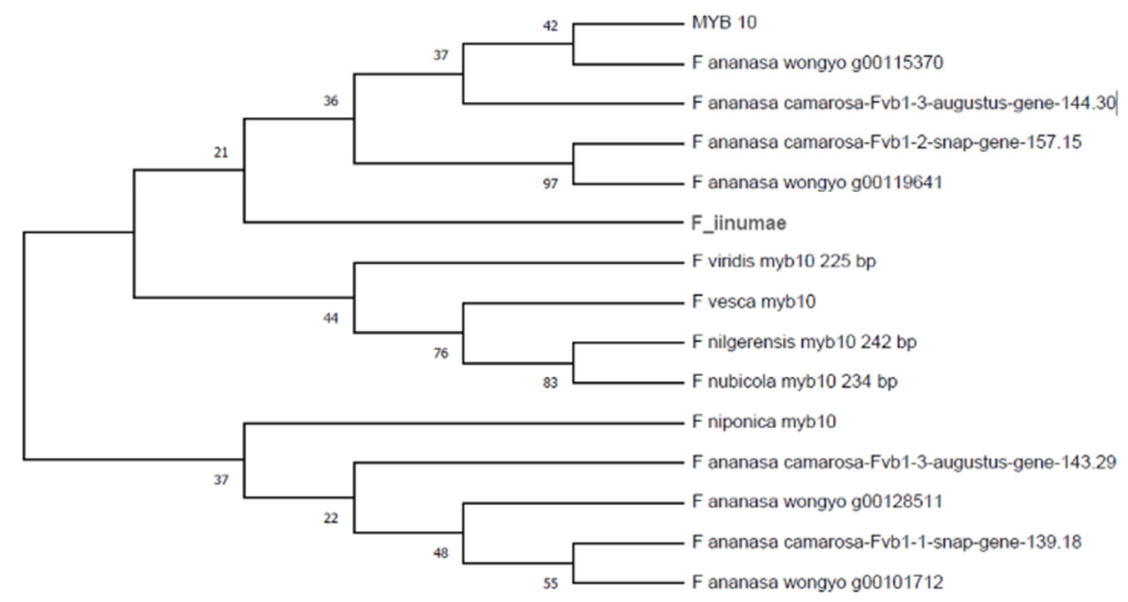

Figure 2. Multiple protein sequence alignment of Fragaria MYB10: (A) the conserved motif A [ANDV] and motif B [RPRPRTF] are highlighted; the phylogenetic relationship between MYB10 transcription factors in Fragaria (B).

\subsection{Relative Expression Analysis of MYB10 and Anthocyanin Biosynthesis Genes}

In order to gain insight into the MYB10 mediated regulation of anthocyanin biosynthesis, gene expression in mature flesh tissue of four cultivars with varying fruit colors was investigated (Figure 4). In a similar pattern with the RNA-seq results, the structural genes PAL1 (g00011621), CHI2 (g00141293), CHI3 (g00133681), DFR (g00093085), FGT2 (g00146246), and ANS (g00093602) showed higher expression in 'P69' and 'Maehyang' compared to 'Wongyo 3115', which has a white flesh color. Similarly, the expression level of the homeolog MYB10 ( $g 00119641)$ showed correlation with the redness of the fruit flesh, whereas MYB10 ( $g 00115370)$ was not expressed in all cultivars. 


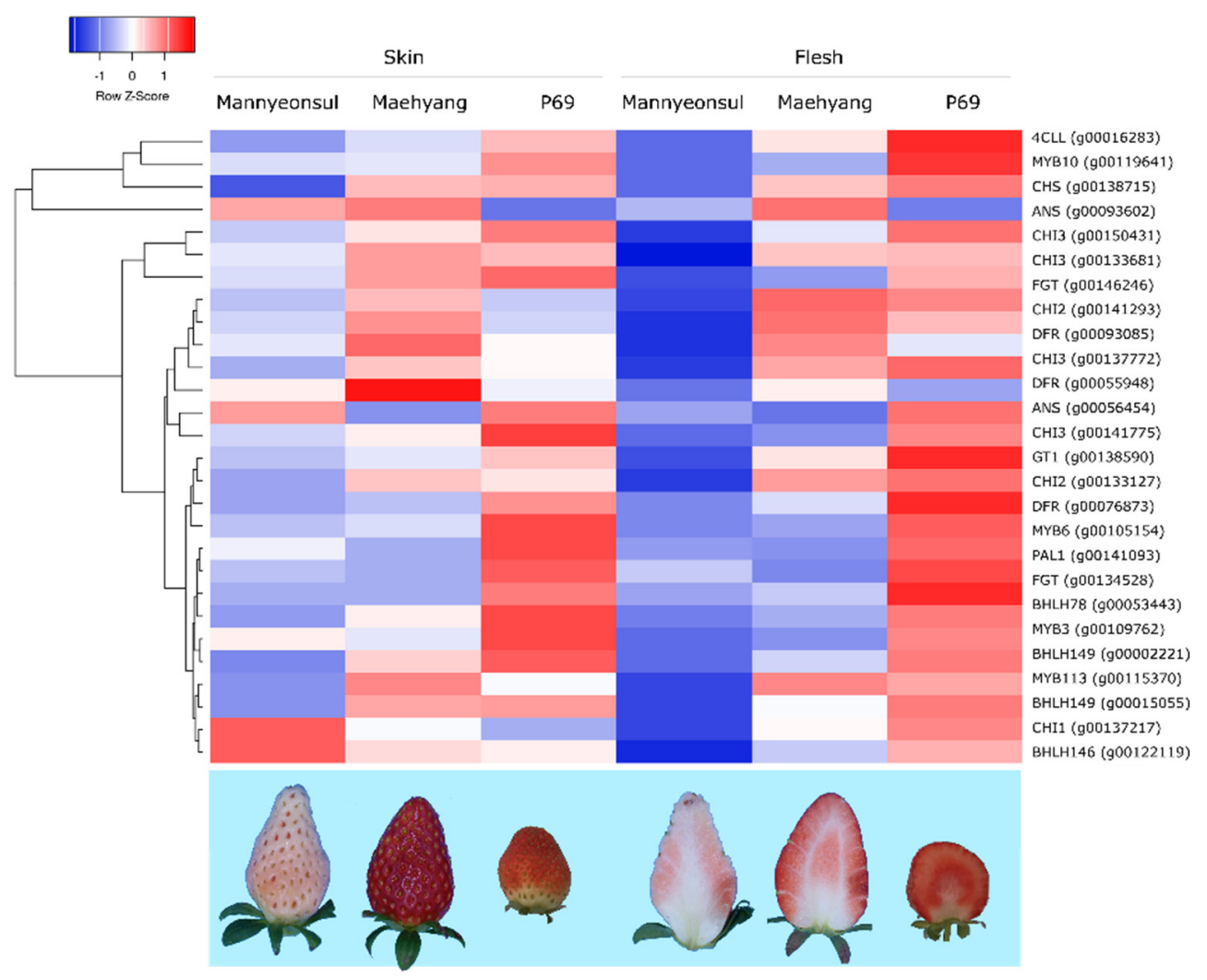

Figure 3. Heatmap displaying the differentially expressed regulatory and structural genes (fold-change $>2$ and FDR $<0.05$ ) involved in the anthocyanin pathway in skin and flesh of light pink and red strawberry varieties in different developmental stages.

\subsection{Identification of QTLs Associated with Fruit Color}

For the QTL analysis associated with the fruit color trait, the phenotyping was performed for two consecutive years (2019-2020). The skin and achene colors were evaluated for the year 2019, while flesh and core color were evaluated additionally for 2020. The maternal line displayed pink skin with white flesh and core color. The paternal line showed red skin with light red flesh and red core color (Figure 5A; Supplementary Dataset S2). Fruit skin color exhibited normal distribution in $\mathrm{F}_{2}$ progenies, while achene color and flesh color biased to red and white, respectively (Figure 5B). QTLs controlling fruit color were analyzed using bin map of 'BS $F_{2}$ (II)' [12] and the color-related traits evaluated for 2019 and 2020. QTLs detected on the common physical position in 'Wongyo 3115' genome were combined to one. A total of seven combined QTLs were detected on chromosomes 1-2, 1-4, 2-1, 6-4 and 7-4 (Table 2). Col_1-2 detected on chromosome 1-2 and could explain all fruit color-related traits with $10-76 \%$ of phenotypic variation. Col_6-4_2 was identified to control skin color for 2019 and achene color for 2020. Other QTLs were only detected for one trait evaluated in one year. 

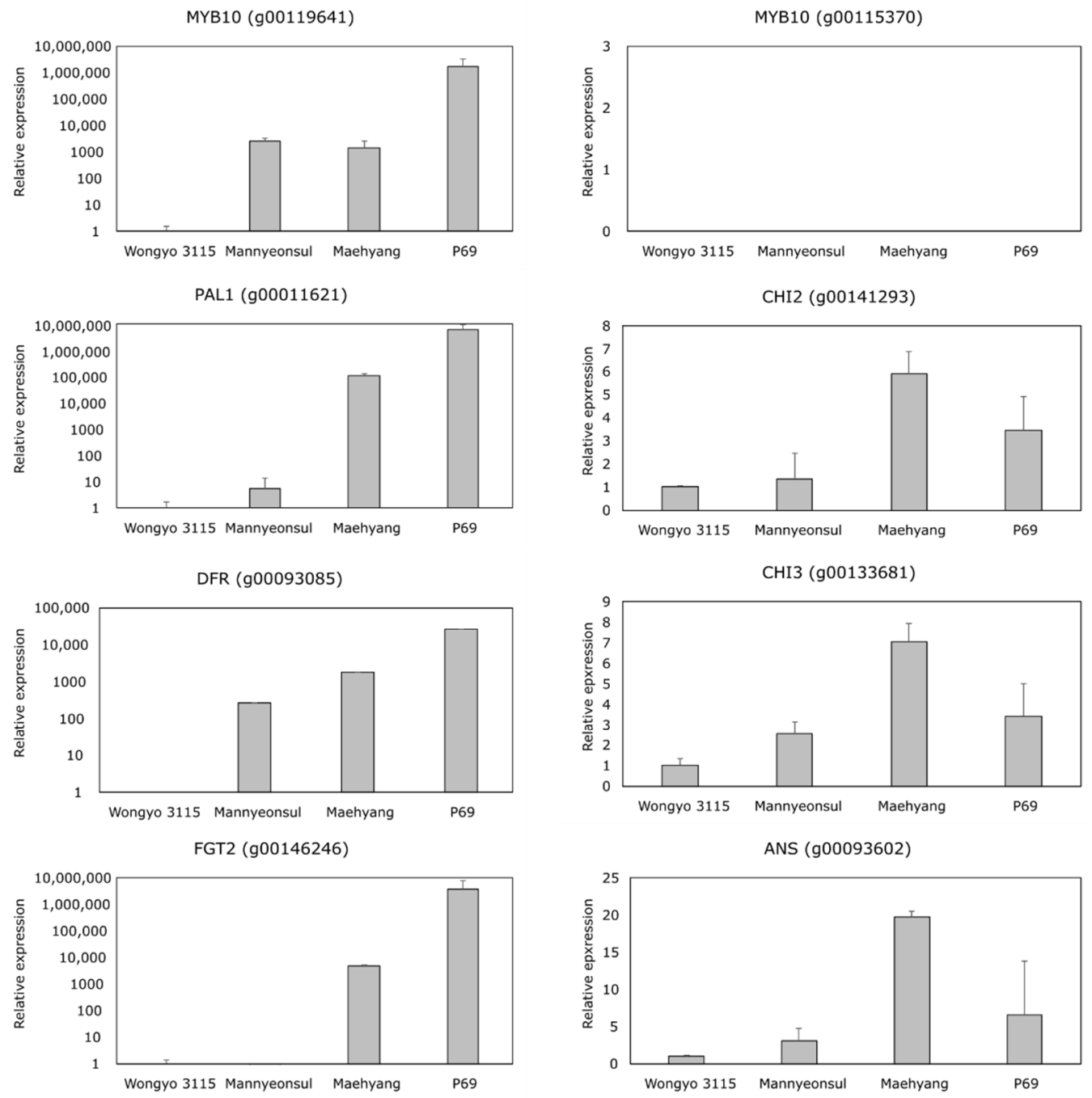

Figure 4. Relative expression analysis of the vital genes involved in the anthocyanin pathway using real-time PCR. The values are relative expression level compared to 'Wongyo 3115' and standard deviation.
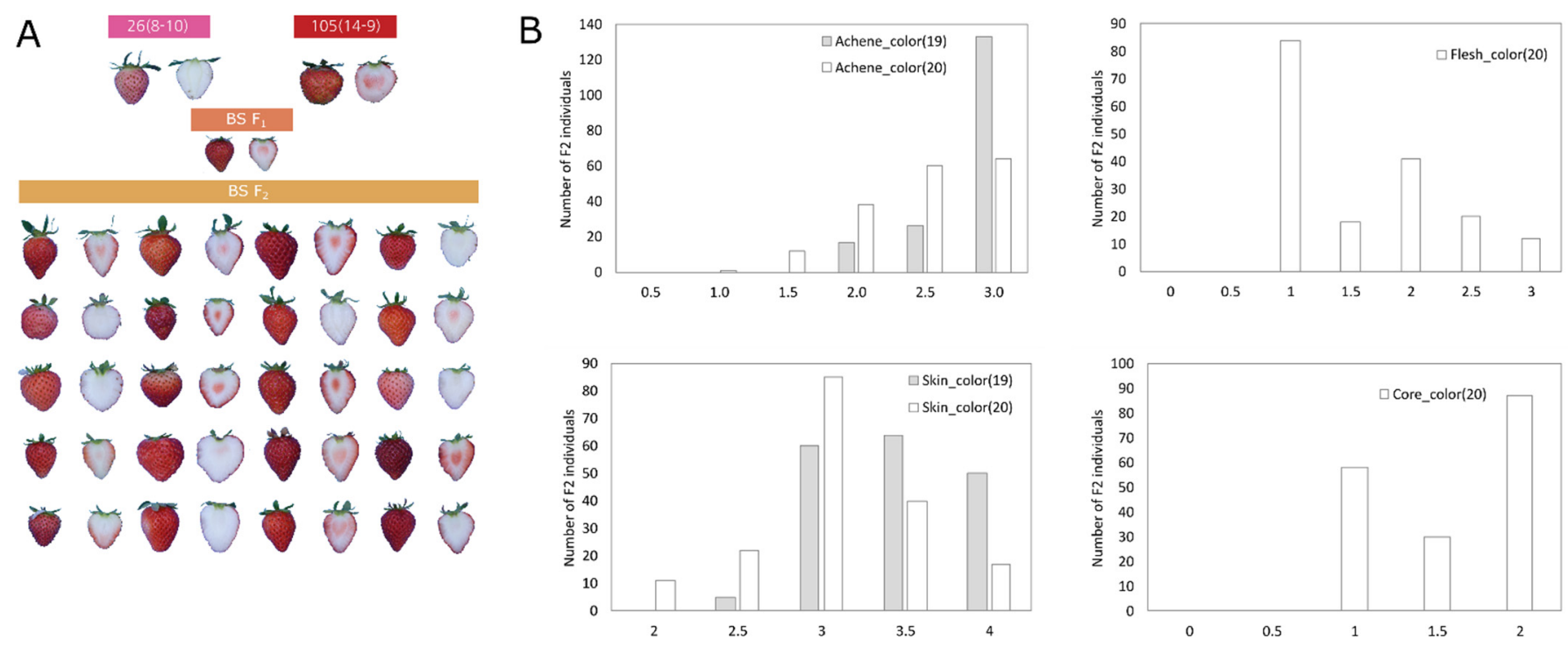

Figure 5. (A) Phenotype of 'BS $F_{2}$ (II)' fruits utilized for the fruit color QTL identification evaluated for 2019 and 2020. (B) Distribution of fruit color related traits in 'BS $F_{2}$ (II)'. 
Table 2. QTLs for fruit color detected in the 'BS $\mathrm{F}_{2}$ (II)' population.

\begin{tabular}{|c|c|c|c|c|c|c|c|c|}
\hline QTL & Chr. & $\begin{array}{l}\text { Physical Position } \\
\text { (Mbp) }\end{array}$ & Trait & $\begin{array}{l}\text { Position } \\
\text { (cM) }\end{array}$ & LOD & $\begin{array}{l}\text { Additive } \\
\text { Effect }{ }^{1}\end{array}$ & $\begin{array}{c}\text { Dominant } \\
\text { Effect }^{2}\end{array}$ & $\begin{array}{l}R^{2} \\
(\%)\end{array}$ \\
\hline \multirow{6}{*}{ Col_1-2 } & \multirow{6}{*}{$1-2$} & \multirow{6}{*}{$12.1-24.5$} & Flesh_color (2020) & $79.1-124.5$ & 28.77 & 0.68 & -0.35 & 75.94 \\
\hline & & & Achene_color (2020) & $81.2-88.2$ & 4.51 & 0.12 & -0.45 & 29.78 \\
\hline & & & Core_color (2020) & $82.2-142.9$ & 32.02 & 0.41 & 0.30 & 38.48 \\
\hline & & & Achene_color (2020) & $89.2-111.5$ & 4.40 & -0.17 & -0.43 & 10.00 \\
\hline & & & Skin_color (2019) & $97.5-142.9$ & 7.19 & 0.23 & 0.18 & 12.91 \\
\hline & & & Skin_color (2020) & $97.5-142.9$ & 11.49 & 0.45 & 0.23 & 20.19 \\
\hline Col_1-4 & $1-4$ & $11.8-15.9$ & Achene_color (2020) & $53.4-66.6$ & 5.67 & -0.22 & -0.10 & 10.04 \\
\hline Col_2-1_1 & $2-1$ & $18.8-20.8$ & Flesh_color (2020) & $74.5-100.4$ & 4.45 & 0.91 & -0.89 & 55.68 \\
\hline Col_2-1_2 & $2-1$ & $21.2-26.9$ & Flesh_color (2020) & $30.7-47.3$ & 5.52 & -0.28 & -0.07 & 6.06 \\
\hline Col_6-4_1 & $6-4$ & $3.0-6.9$ & Achene_color (2019) & $7.0-17.9$ & 3.97 & -0.03 & -0.25 & 5.67 \\
\hline \multirow{2}{*}{ Col_6-4_2 } & \multirow{2}{*}{$6-4$} & \multirow{2}{*}{$15.5-29.7$} & Skin_color (2019) & $127.7-143.7$ & 5.00 & 0.30 & -0.25 & 0.62 \\
\hline & & & Achene_color (2020) & $138.7-144.2$ & 4.17 & -0.26 & 0.11 & 15.09 \\
\hline Col_7-4 & $7-4$ & $17.8-21.8$ & Achene_color (2020) & $58.5-68.1$ & 4.49 & -0.18 & -0.05 & 6.50 \\
\hline
\end{tabular}

\subsection{Identification of Predicted Candidate Genes Associated with Fruit Color Using QTL and RNA-Seq Analysis}

The QTL information and 'Wongyo 3115' annotation data were employed for the prediction of candidate genes associated with fruit color. To investigate the candidate genes associated with fruit color, genes that displayed differential expression between matured fruits of pink color 'Mannyeonsul', and red color 'Maehyang', and 'P69' located in QTL regions were selected. The QTL regions covered a total of 1605 differentially expressed genes based on the physical position details retrieved from the 'Wongyo 3115' annotation data (Table S3). Among the QTL regions, Col_6-4 consisted of a higher number of genes (686) followed by Col_2-1 (281 genes).

The paired analysis between two inbred lines was performed with the filtering criteria of two-fold-change difference. The number of differentially expressed genes in only skin and flesh were 386 and 394, respectively. In contrast, a total of 825 genes showed a difference in the expression in both tissues. Further, the differentially expressed genes located in the QTL regions were extracted based on the physical position from the annotation data of the 'Wongyo 3115' assembly. A total of 11 potential candidate genes including vital structural and regulatory genes associated with fruit color were identified by utilizing the transcriptome and QTL data (Table 3; Figure 6, Table S4). In particular, we identified the DFR, ANS, CHI3, GT1, and MYB10 transcription factors in the QTL regions, which play a vital role in the determination of fruit color in strawberries. In addition, transcriptome analysis indicated the up-regulation of structural and regulatory genes in red fruit bearing 'P69' cultivar than the light pink fruits bearing the 'Mannyeonsul' cultivar. In addition, the QTL regions consisted of several regulatory genes such as MYB (4), bHLH (4), and WD-repeats (4) with potential roles in anthocyanin pathway (Table S3). 
Table 3. Predicted candidate genes in QTLs associated with the fruit color in the 'BS $\mathrm{F}_{2}$ (II)' population.

\begin{tabular}{|c|c|c|c|c|}
\hline QTL & Gene ID & Chr. & Description & Function \\
\hline Col_6-4_1 & g00015421 & $6-4$ & $\begin{array}{l}\text { DFR: Bifunctional dihydroflavonol } \\
\text { 4-reductase/flavanone 4-reductase }\end{array}$ & $\begin{array}{l}\text { Anthocyanin biosynthesis/Secondary } \\
\text { metabolism }\end{array}$ \\
\hline Col_6-4_2 & g00017208 & $6-4$ & ANS1: Leucoanthocyanidin dioxygenase 1 & $\begin{array}{l}\text { Anthocyanin biosynthesis/Secondary } \\
\text { metabolism }\end{array}$ \\
\hline Col_6-4_2 & g00018684 & $6-4$ & $\mathrm{C} 1$ : Anthocyanin regulatory $\mathrm{C} 1$ protein & Anthocyanin biosynthesis \\
\hline Col_7-4 & g00137772 & $7-4$ & CHI3: Probable chalcone-flavonone isomerase 3 & $\begin{array}{l}\text { Anthocyanin biosynthesis/Secondary } \\
\text { metabolism }\end{array}$ \\
\hline Col_7-4 & g00138590 & $7-4$ & GT1: Anthocyanidin 3-O-glucosyltransferase 1 & $\begin{array}{l}\text { Anthocyanin biosynthesis/Secondary } \\
\text { metabolism }\end{array}$ \\
\hline Col_1-2 & g00119641 & $1-2$ & MYB113: Transcription factor MYB113/MYB10 & DNA binding/Secondary metabolism \\
\hline Col_1-4 & g00128511 & $1-4$ & Similar to PNS1: Protein PNS1/MYB10 & DNA binding/Secondary metabolism \\
\hline Col_7-4 & g00138251 & $7-4$ & UGT88B1: UDP-glycosyltransferase 88B1 & \\
\hline Col_6-4_2 & g00018719 & $6-4$ & CYP750A1: Cytochrome P450 750A1 & ABA biosynthesis \\
\hline Col_6-4_2 & g00019239 & $6-4$ & $\begin{array}{l}\text { GT6: UDP-glucose flavonoid } \\
\text { 3-O-glucosyltransferase } 6\end{array}$ & $\begin{array}{l}\text { Anthocyanin biosynthesis/Secondary } \\
\text { metabolism }\end{array}$ \\
\hline Col_6-4_2 & g00018681 & $6-4$ & GPI: Glucose-6-phosphate isomerase & Sugar metabolism \\
\hline
\end{tabular}

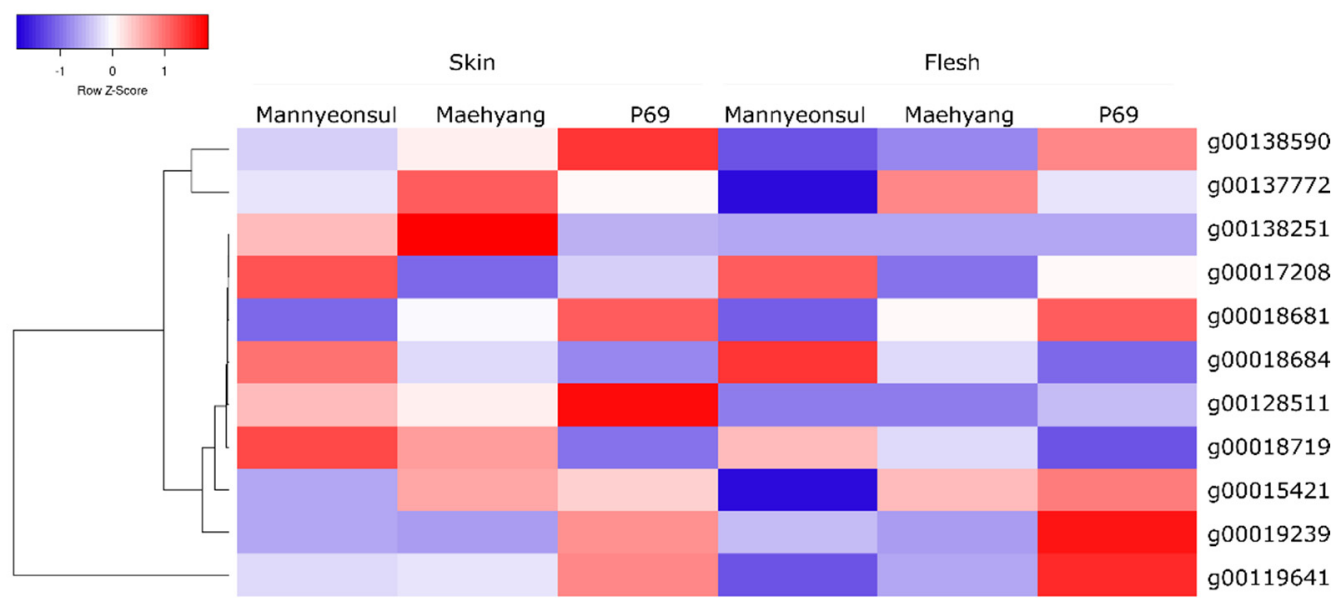

Figure 6. Heat map representation of 11 differentially expressed predicted candidate genes (fold-change $>2$ and FDR $<$ 0.05 ) associated with fruit color in three cultivars of strawberry.

\section{Discussion}

Strawberry is a widely cultivated horticultural crop with tremendous nutritional and commercial importance. The aesthetic and nutraceutical values of strawberry renders several benefits to human health [3]. The fruit colors of strawberry are determined by the distribution and accumulation of water-soluble, flavonoid based pigments called anthocyanin. Based on the anthocyanin contents the strawberry fruits are observed in different colors from white to dark red. The anthocyanin levels significantly increase during the development of fruits and reaches higher levels at the ripening stage [19]. Recent reports suggested the predominant role of MYB10 transcription factor in the determination of fruit color in strawberry $[9,10,20]$. Therefore, in the present endeavor, we have performed genome-wide investigation of MYB10 transcription factor in diploid and octoploid strawberry.

For the genome-wide analysis, a total of eight genomes including six diploids and two octoploids were investigated. A sequence-based homology search using the $F . \times$ ananassa MYB10 resulted in the 14 MYB10 genes in the genomes. All the diploids consisted of one 
MYB10, whereas the octoploids possessed four homeologs of MYB10. In all the genomes, the MYB10 loci were observed in chromosome 1. In general, the gene structure of MYB10 encompassed three exons and two introns [10]. In Fragaria, all diploids consisted of three exons separated by two introns whereas in octoploids the MYB10 homeologs g00128511 and 143.29 displayed two exons and one intron. On the other hand, the homeolog in $F$. $\times$ ananassa 'Camarosa' 139.18 possessed six exons spaced by five introns and $F . \times$ ananassa 'Wongyo 3115' g00101712 displayed only one exon. Duplication events in the genome of octoploid strawberry resulted in four homeologs MYB10 in the homozygous 'Wongyo 3115' genome. MYB10 was identified in each subgenomes; however, in the heterozygous 'Camarosa', Ch1-3 consisted of two MYB10 homologs and none was identified on Ch1-4. Genome doubling and duplication events in the genome of octoploid strawberry led to the occurrence of multiple MYB10 homeologs in comparison with diploids.

Despite the presence of multiple homeologs, the MYB10 homeologs in 'Wongyo 3115' ( $g 00119641$ and $g 00115370$ ) displayed significant expression in the strawberry fruits employed in this study. In addition, MYB10 (g00119641) contributed by F. iinumae subgenome of octoploid strawberry displayed higher expression in comparison with g00115370. According to Fu et al. [21], the homeologous gene expression in allopolyploid crops exhibit spatial, temporal, and complex features that also tend to result from alternative splicing. The differential gene expression in polyploid results in functional plasticity and incorporates novel phenotypes that permit the adaptability of polyploid more efficiently to environmental fluctuations [21]. Moreover, our results are in accordance with the recent report [10] suggesting the anthocyanin biosynthesis regulation is mediated by the MYB10 homeolog contributed by the F. iinumae subgenome of octoploid strawberry. The disruption of MYB10 function could be attributed to the difference in the gene structure, occurrence of repeats in the functional regions, and premature codon displacement; these factors could have led to the mere expression or non-expression of other MYB10 homeologs in octoploid strawberry.

Anthocyanin biosynthesis and accumulation in plants are determined by the phenylpropanoid pathway. The expression of major structural genes such as PAL, GT, FGT, 4CLL, $C H I$, and $C H S$ in synergistic expression correlation with regulatory genes like $M Y B, b H L H$, and $W D-40$ repeats facilitate the phenotypic variations in the anthocyanin distribution in strawberry [10]. Therefore, to investigate the expression of vital structural and regulatory genes involved in anthocyanin biosynthesis, the transcriptome analysis in three cultivars of strawberry such as 'Mannyeonsul' (light pink fruits), 'Maehyang' (red fruits), and 'P69' (dark red fruits) were conducted. The results revealed a higher expression of $P A L$ (g00141093), GT (g00138590), FGT (g00134528), 4CLL (g00016283), CHI3 (g00150431), CHI2 (g00133127), and CHS (g00138715) in the dark red fruit bearing 'P69' cultivar and lower expression in light pink fruits of 'Mannyeonsul'. Similarly, the transcriptome levels of MYB10 correlated with expression of structural genes. MYB10 transcription factor acts as one of the important factors that influence anthocyanin biosynthesis pathway in both diploid $F$. vesca [22] and octoploid F. $\times$ ananassa [10]. According to Lin-Wang et al. [23], the RNAi based silencing of $M Y B 10$ correlated with the decreased in the expression levels vital genes such as CHI, CHS, F3H, and DFR responsible for anthocyanin synthesis. Nevertheless, Lin et al. [24] reported that the MYB10 gene displayed non-significant difference in expression between red and white fleshed strawberry. In addition, Medina-Puche et al. [25] evidenced that the gene ANS is not regulated by transient silencing of FaMYB10 in cultivated strawberry. These studies suggest that the expression of MYB10 acts as an additional factor in the regulation of vital structural genes involved in the anthocyanin biosynthesis pathway in strawberry. Moreover, MYB10 also influenced the expression of other regulatory genes such as $b H L H 33, M Y B 89$, and MYB1 [23]. Similarly, the relative expression of the structural genes displayed a positive relation with the expression of MYB10 (g00119641).

Consequently, the RNA-seq data confirmed the positive regulation of MYB10 transcription factor and the corresponding structural genes in the dark red matured fruits of 'P69'. The stage specific relative expression analysis using qPCR suggested that the 
expression of $M Y B 10$ as well as the vital structural genes are increased during the ripening stage in all the cultivars. However, a higher fold increase has been observed in the skin and flesh tissues of dark red fruit bearing 'P69' cultivar in comparison with other cultivars. Several reports have evidenced the positive relationship of the MYB10 regulatory gene with structural genes involved in early and late anthocyanin biosynthesis in various crops such as apple [26], pear [27], sweet cherry [28], and peach [29]. Recently, Wang et al. [30], reported an 8-bp insertion in the MYB10 coding region which correlated with the color loss in octoploid strawberry cultivar 'Snow Princess'. This study was further evidenced by Castillejo et al. [10], illustrating the allelic variation in the MYB10 determine the color variation in skin and flesh tissues of strawberry. Similarly, in F. vesca 'Yellow Wonder', a single nucleotide mutation in the 35th position of MYB10 sequence encoding the R2 DNA binding domain resulted in yellow color fruits [22].

In this QTL study, seven QTLs controlling fruit colors were detected (Table 3) and two QTLs (Col_1-2 and Col_6-4_2) control multiple traits. For QTL Col_1-2, $\mathrm{F}_{2}$ individuals with maternal genotype showed darker skin, flesh, and core color than those of paternal genotype. Conversely, paternal genotyped individuals showed darker achene color. Col_6$4 \_2$ also showed dissimilar pattern for skin and achene color. In a similar manner, achene color was negatively correlated with other fruit color related traits whereas the other traits were positively correlated each other (Table S5). '8-10' and 'Mannyeonsul' showed pink skin and red achene while '105 (14-9)' and 'P69' showed dark red skin and yellowish achene. In previous research, hormone accumulation and gene expression related to fruit development showed inconsistency in achene and receptacle [31,32]. From these findings, we assume that achene development and ripening might be controlled by different a mechanism from the receptacle. MYB10 was proposed as a strong candidate for achene, skin, flesh, and core color of fruits. QTLs controlling skin color parameter a and $\mathrm{b}$ detected on LGIa [13], and parameter a detected on LG1.3 [17], were also located on Chr1-2 linked to the MYB10 gene. Gene expression analysis support the MYB10 mediated regulation of structural genes which influence the accumulation of anthocyanin in fruit tissues of octoploid strawberry cultivars 'Mannyeonsul', 'Maehyang', and 'P69'.

The 'Wongyo 3115' annotation information and QTL information also facilitated the prediction of potential candidate genes that could influence the skin and flesh colors in octoploid strawberries. These candidate genes participate in several essential processes such as sugar metabolism, secondary metabolism, auxin metabolism, hormone biosynthesis, and DNA binding. Potential genes such as DFR, ANS1, CHI3, GT1, UGT, and MYB10 with an active role in anthocyanin biosynthesis have been identified as candidate genes in Col_6-4_1, Col_6-4_2,Col_7-4, and Col_1-2 QTL regions.

The identification of candidate genes influencing the anthocyanin pathway can enhance the tailoring of the pathway to acquire strawberry fruits with varying colors. The importance of the candidate genes such as DFR, ANS1, CHI3, GT1, UGT, and MYB10 in the biosynthesis of anthocyanin have been evidenced in several horticultural crops. Particularly, in cultivated strawberry, the fruit color is vital for the consumer preference and therefore considered as a vital trait for strawberry breeding. Similarly, we have identified transcription factors belonging to MYB, bHLH, and WD families in the QTL regions. Several researchers have asserted the important functions of transcription factors and hormones in strawberry fruit development $[31,33,34]$. In future endeavors, the vital role of TFs associated with the fruit color can be attained and the present QTL data can facilitate the process. Overall, the outcomes of the present study provide a valuable resource for future functional characterization and development of trait specific markers associated with MYB10 for breeding new cultivars of strawberry with diverse fruit color.

\section{Materials and Methods}

\subsection{Plant Materials}

To analyze the gene expression related to fruit color, two varieties 'Maehyang' and 'Mannyeonsul', and one inbred line 'P69', were used. 'Maehyang' originated from a cross 
between 'Tochinomine' and 'Akihime' and has red skin, flesh, and core color (registration number 5160, Korea Seed and Variety Service). 'Mannyeonsul' is known as mutant of 'Akihime' and shows pink skin and flesh color (registration number 7867, Korea Seed and Variety Service). 'P69' is an inbred line derived from 'Benihoppe' by nine self-pollination and has red skin, flesh, and core color. A total of 186 'BS $\mathrm{F}_{2}(\mathrm{II})^{\prime}$ ' population derived from a cross between '8-10' and '105 (14-9)' were used for QTL analysis. All plants were grown in raised beds located in Wanju, Republic of Korea. 'BS $\mathrm{F}_{2}$ (II)' was grown for two consecutive years (2019 and 2020) and four plants per $F_{2}$ line were planted for phenotyping.

\subsection{Identification of MYB10 Transcription Factors in Fragaria}

The genomic resources of six diploid Fragaria species and octoploid $F . \times$ annanasa 'Camarosa' were downloaded from the GDR database (https: / / www.rosaceae.org/species / fragaria/all (accessed on 2 June 2021)) and $F . \times$ annanasa 'Wongyo 3115' from GenBank (https: / / www.ncbi.nlm.nih.gov / assembly /GCA_019022445.1 (accessed on 25 June 2021)). For the identification of MYB10 transcription factor, homology sequence search using BLAST tool, with the query MYB10 transcription factor (QIZ03070) from $F . \times$ ananassa, was employed. The sequences with 100 to $98 \%$ similarity were selected for further analysis. The location of MYB10 sequences were extracted from the genome feature file (gff) for each genome. Multiple sequence alignments of the MYB10 protein sequences were performed using Clustal X (version 2.1) in multiple alignment mode. A maximum likelihood phylogenetic tree was constructed using MEGA 6.0 with the following parameters: 1000 bootstrap replicates, pairwise alignment, Dayhoff model, Gamma distributed (G), 2 Gamma parameters, and pairwise deletion.

\subsection{Analysis of Conserved Motifs, Gene Structures, and Cis-Elements}

The MYB10 protein sequences were analyzed with the MEME (https:/ / meme-suite. org/meme/ (accessed on 20 May 2021)) online software to identify the occurrence of conserved motifs by employing the following parameters: normal mode, any number of repetitions, and two motifs. Analysis of the MYB10 gene structure, including the intron and exons, was acquired from the Fragaria genome annotations available in rosaceae database (https: / /www.rosaceae.org (accessed on 20 May 2021)) and visualized using Gene Structure Server version 2.0 (http:/ / gsds.cbi.pku.edu.cn/ (accessed on 20 May 2021)). For cis-element analysis, the $2 \mathrm{~kb}$ upstream transcriptional start site of each MYB10 from diploids and octoploids was investigated using Plant Care (http://bioinformatics.psb. ugent.be/webtools/plantcare/html/ (accessed on 7 July 2021)) tool.

\subsection{RNA-Seq Analysis of Skin and Flesh}

Skin color of three cultivars 'Mannyeonsul', 'Maehyang', and 'P69' were measured by colorimeter (CR-400, Minolta, Japan) at mature stage. The skin and flesh tissues were separated from matured fruit samples using a scalpel according to Sánchez-Sevilla et al., [32]. Three biological replicates were employed for transcriptome analysis. The samples were homogenized and the total RNA was extracted using Trizol (Invitrogen, Waltham, MA, USA) according to the manufacturer's protocol. After the quality evaluation, cDNA libraries were prepared from the RNA samples and paired-end library was constructed using the Truseq stranded mRNA Prep kit (Illumina Inc., San Diego, CA, USA) according to the manufacturer's instructions. After purification, the sequencing library was produced by PCR amplification and sequenced using the Novaseq6000 platform (Illumina Inc., San Diego, CA, USA). The raw reads with low quality and the clean reads were then assembled and mapped to the 'Wongyo 3115' reference genome (Genebank accession number: JACXYW000000000) using the Top hat v2.0.13. The differential expression was analyzed using the cuffdiff v2.2.0. Genes with the FPKM estimate 2-fold higher than that of the lowest one were identified as differentially expressed genes (DEGs). Gene expression differences were validated using a chi-square test and false discovery rate (FDR). Genes with an FDR $<0.001$, and for which the FPKM estimate was 2-fold higher than that of the 
lowest one, were identified as DEGs. The functional annotations were performed using DAVID 6.8 Beta. A heat map was generated using significantly altered genes in fruits of both cultivars. The raw intensity data (FPKM) were $\log 2$ transformed and then utilized for the calculation of $\mathrm{Z}$ scores.

\subsection{RT-PCR Analysis}

The flesh tissues of mature fruits were separated from 'Wongyo 3115', 'Mannyeonsul', 'Maehyang', and 'P69'. RNA isolation was performed in similar manner with transcriptome analysis as mentioned in the previous section. The cDNA biosynthesis was performed using a Quantitect Reverse Transcription Kit (Qiagen, Hilden, Germany) and RT-PCR was performed as described previously [35]. Gene expression level was calculated relatively to 'Wongyo 3115'. All primers were designed based on the annotation data of 'Wongyo 3115' reference genome and are listed in Table S6.

\subsection{QTL Analysis and Identification of Candidate Genes}

The fruit color related traits of the 'BS $F_{2}$ (II)' population were evaluated as two to three scales (Table S2). QTL analysis was performed using the phenotypes of 2019 and 2020 and genetic map constructed by Lee and Manivannan et al. [12]. Composite interval mapping of Windows QTL Cartographer 2.5 [36] with default option was used for QTL analysis. By using the 500 times permutation test ( $p$-value $<0.05$ ), significant QTLs exceeding the LOD threshold were selected. Based on the physical position of QTLs in 'Wongyo 3115' genome, overlapped QTLs were combined to one QTL and named based on the sub-chromosome number. For example, six QTLs were detected on chromosome 1-2 and combined to one QTL named 'Col_1-2' (Table 2). By comparison of QTL analysis and RNA-Seq results, candidate genes were selected. In detail, genes expressed differentially with respect to 'Maehyang' or 'P69' in comparison with 'Mannyeonsul' and located in the QTL region were considered as candidate genes for fruit color.

Supplementary Materials: The following are available online at https:/ / www.mdpi.com/article/10 .3390/ijms222212587/s1.

Author Contributions: Conceptualization, A.M., K.H. and D.-S.K.; methodology, A.M., K.H., J.P.H. and J.K.; investigation, S.Y.L. and H.-E.L.; resources, S.Y.L. and Y.-R.L.; writing-original draft preparation, A.M. and K.H.; writing-review and editing, A.M., K.H., H.-E.L. and E.S.L.; visualization, A.M., K.H. and J.P.H.; supervision, D.-S.K. All authors have read and agreed to the published version of the manuscript.

Funding: This work was carried out with the support of "Cooperative Research Program for Agriculture Science and Technology Development (Project No: PJ01416902)", Rural Development Administration, Republic of Korea. A.M. and Y.R.L. were supported by the RDA Research Associate Fellowship Program of National Institute of Horticultural and Herbal Science, Rural Development Administration, Republic of Korea.

Data Availability Statement: Whole-genome sequence data of 'Wongyo 3115' have been deposited in NCBI under the Bioproject PRJNA662854 and Biosample SAMN16094694 (accession number SRR14102268-SRR14102276). This whole genome shotgun project has been deposited at GenBank under the accession JACXYW000000000. The version described in this paper is JACXYW010000000.

Conflicts of Interest: The authors declare no conflict of interest.

\section{References}

1. Pabo, C.O.; Sauer, R.T. Transcription factors: Structural families and principles of DNA recognition. Annu. Rev. Biochem. 1992, 61, 1053-1095. [CrossRef] [PubMed]

2. Riechmann, J.L.; Heard, J.; Martin, G.; Reuber, L.; Jiang, C.-Z.; Keddie, J.; Adam, L.; Pineda, O.; Ratcliffe, O.J.; Samaha, R.R.; et al. Arabidopsis Transcription Factors: Genome-Wide Comparative Analysis Among Eukaryotes. Science 2000, 290, $2105-2110$. [CrossRef] [PubMed] 
3. Pillet, J.; Yu, H.-W.; Chambers, A.H.; Whitaker, V.M.; Folta, K.M. Identification of candidate flavonoid pathway genes using transcriptome correlation network analysis in ripe strawberry (Fragaria $\times$ ananassa) fruits. J. Exp. Bot. 2015, 66, 4455-4467. [CrossRef]

4. Zhang, Y.; Butelli, E.; Martin, C. Engineering anthocyanin biosynthesis in plants. Curr. Opin. Plant Biol. 2014, 19, 81-90. [CrossRef] [PubMed]

5. Petroni, K.; Tonelli, C. Recent advances on the regulation of anthocyanin synthesis in reproductive organs. Plant Sci. 2011, 181, 219-229. [CrossRef]

6. Wang, N.; Qu, C.; Jiang, S.; Chen, Z.; Xu, H.; Fang, H.; Su, M.; Zhang, J.; Wang, Y.; Liu, W.; et al. The proanthocyanidin specific transcription factor Md MYBPA 1 initiates anthocyanin synthesis under low temperature conditions in red-fleshed apples. Plant J. 2018, 96, 39-55. [CrossRef]

7. Hichri, I.; Barrieu, F.; Bogs, J.; Kappel, C.; Delrot, S.; Lauvergeat, V. Recent advances in the transcriptional regulation of the flavonoid biosynthetic pathway. J. Exp. Bot. 2011, 62, 2465-2483. [CrossRef]

8. Stracke, R.; Werber, M.; Weisshaar, B. The R2R3-MYB gene family in Arabidopsis thaliana. Curr. Opin. Plant Biol. 2001, 4, 447-456. [CrossRef]

9. Zhang, J.; Lei, Y.; Wang, B.; Li, S.; Yu, S.; Wang, Y.; Li, H.; Liu, Y.; Ma, Y.; Dai, H.; et al. The high-quality genome of diploid strawberry (Fragaria nilgerrensis) provides new insights into anthocyanin accumulation. Plant Biotechnol. J. 2020, 18, 1908-1924. [CrossRef]

10. Castillejo, C.; Waurich, V.; Wagner, H.; Ramos, R.; Oiza, N.; Muñoz, P.; Triviño, J.C.; Caruana, J.; Liu, Z.; Cobo, N.; et al. Allelic Variation of MYB10 Is the Major Force Controlling Natural Variation in Skin and Flesh Color in Strawberry (Fragaria spp.) Fruit. Plant Cell 2020, 32, 3723-3749. [CrossRef]

11. Edger, P.P.; Poorten, T.J.; VanBuren, R.; Hardigan, M.A.; Colle, M.; McKain, M.R.; Smith, R.D.; Teresi, S.J.; Nelson, A.D.L.; Wai, C.M.; et al. Origin and evolution of the octoploid strawberry genome. Nat. Genet. 2019, 51, 541-547. [CrossRef]

12. Lee, H.-E.; Manivannan, A.; Lee, S.Y.; Han, K.; Yeum, J.-G.; Jo, J.; Kim, J.; Rho, I.R.; Lee, Y.-R.; Lee, E.S.; et al. Chromosome Level Assembly of Homozygous Inbred Line ‘Wongyo 3115’ Facilitates the Construction of a High-Density Linkage Map and Identification of QTLs Associated with Fruit Firmness in Octoploid Strawberry (Fragaria $\times$ ananassa). Front. Plant Sci. 2021, 12, 1337. [CrossRef] [PubMed]

13. Lerceteau-Köhler, E.; Moing, A.; Guérin, G.; Renaud, C.; Petit, A.; Rothan, C.; Denoyes, B. Genetic dissection of fruit quality traits in the octoploid cultivated strawberry highlights the role of homoeo-QTL in their control. Theor. Appl. Genet. 2012, 124, 1059-1077. [CrossRef]

14. Labadie, M.; Vallin, G.; Petit, A.; Ring, L.; Hoffmann, T.; Gaston, A.; Potier, A.; Schwab, W.G.; Rothan, C.; Denoyes, B. Metabolite Quantitative Trait Loci for Flavonoids Provide New Insights into the Genetic Architecture of Strawberry (Fragaria $\times$ ananassa) Fruit Quality. J. Agric. Food Chem. 2020, 68, 6927-6939. [CrossRef] [PubMed]

15. Jassmine, Z.; Cabeza, A.; Domínguez, P.; Medina, J.J.; Valpuesta, V.; Denoyes, B.; Sevilla, J.F.S.; Amaya, I. Quantitative trait loci and underlying candidate genes controlling agronomical and fruit quality traits in octoploid strawberry (Fragaria $\times$ ananassa). Theor. Appl. Genet. 2011, 123, 755-778. [CrossRef]

16. Pott, D.M.; Vallarino, J.G.; Cruz-Rus, E.; Willmitzer, L.; Sánchez-Sevilla, J.F.; Amaya, I.; Osorio, S. Genetic analysis of phenylpropanoids and antioxidant capacity in strawberry fruit reveals mQTL hotspots and candidate genes. Sci. Rep. 2020, $10,20197$. [CrossRef] [PubMed]

17. Alarfaj, R.; El-Soda, M.; Antanaviciute, L.; Vickerstaff, R.; Hand, P.; Harrison, R.J.; Wagstaff, C. Mapping QTL under-lying fruit quality traits in an F1 strawberry population. J. Hortic. Sci. Biotechnol. 2021, 96, 634-645. [CrossRef]

18. Lin-Wang, K.; Bolitho, K.; Grafton, K.; Kortstee, A.; Karunairetnam, S.; McGhie, T.K.; Espley, R.V.; Hellens, R.P.; Allan, A.C. An R2R3 MYB transcription factor associated with regulation of the anthocyanin biosynthetic pathway in Rosaceae. BMC Plant Biol. 2010, 10, 50. [CrossRef]

19. Parra-Palma, C.; Morales-Quintana, L.; Ramos, P. Phenolic Content, Color Development, and Pigment-Related Gene Expression: A Comparative Analysis in Different Cultivars of Strawberry during the Ripening Process. Agronomy 2020, 10, 588. [CrossRef]

20. Chen, G.; Xu, P.; Pan, J.; Li, Y.; Zhou, J.; Kuang, H.; Lian, H. Inhibition of FvMYB10 transcriptional activity promotes color loss in strawberry fruit. Plant Sci. 2020, 298, 110578. [CrossRef]

21. Fu, D.; Mason, A.S.; Xiao, M.; Yan, H. Effects of genome structure variation, homeologous genes and repetitive DNA on polyploid crop research in the age of genomics. Plant Sci. 2016, 242, 37-46. [CrossRef]

22. Zhang, J.; Zhang, Y.; Dou, Y.; Li, W.; Wang, S.; Shi, W.; Sun, Y.; Zhang, Z. Single nucleotide mutation in FvMYB10 may lead to the yellow fruit in Fragaria vesca. Mol. Breed. 2017, 37, 35. [CrossRef]

23. Lin-Wang, K.; McGhie, T.K.; Wang, M.; Liu, Y.; Warren, B.; Storey, R.; Espley, R.V.; Allan, A.C. Engineering the anthocyanin regulatory complex of strawberry (Fragaria vesca). Front. Plant Sci. 2014, 5, 651. [CrossRef]

24. Lin, Y.; Jiang, L.; Chen, Q.; Li, Y.; Zhang, Y.; Luo, Y.; Zhang, Y.; Sun, B.; Wang, X.; Tang, H. Comparative Transcriptome Profiling Analysis of Red- and White-Fleshed Strawberry (Fragaria $\times$ ananassa) Provides New Insight into the Regulation of the Anthocyanin Pathway. Plant Cell Physiol. 2018, 59, 1844-1859. [CrossRef] [PubMed]

25. Medina-Puche, L.; Cumplido-Laso, G.; Amil-Ruiz, F.; Hoffmann, T.; Ring, L.; Rodrguez-Franco, A.; Caballero, J.L.; Schwab, W.; Muoz-Blanco, J.; Blanco-Portales, R. MYB10 plays a major role in the regulation of flavonoid/phenylpropanoid metabolism during ripening of Fragaria $\times$ ananassa fruits. J. Exp. Bot. 2014, 65, 401-417. [CrossRef] [PubMed] 
26. Telias, A.; Lin-Wang, K.; Stevenson, D.E.; Cooney, J.M.; Hellens, R.P.; Allan, A.C.; Hoover, E.E.; Bradeen, J.M. Apple skin patterning is associated with differential expression of MYB10. BMC Plant Biol. 2011, 11, 93. [CrossRef] [PubMed]

27. Wang, Y.; Zhang, X.; Zhao, Y.; Yang, J.; He, Y.; Li, G.; Ma, W.; Huang, X.; Su, J. Transcription factor PyHY5 binds to the promoters of PyWD40 and PyMYB10 and regulates its expression in red pear 'Yunhongli No. 1'. Plant Physiol. Biochem. 2020, 154, 665-674. [CrossRef]

28. Starkevič, P.; Paukstyte, J.; Kazanaviciute, V.; Denkovskiene, E.; Stanys, V.; Bendokas, V.; Siksnianas, T.; Razanskiene, A.; Ražanskas, R. Expression and Anthocyanin Biosynthesis-Modulating Potential of Sweet Cherry (Prunus avium L.) MYB10 and bHLH Genes. PLoS ONE 2015, 10, e0126991. [CrossRef]

29. Zhou, H.; Liao, L.; Xu, S.; Ren, F.; Zhao, J.; Ogutu, C.; Wang, L.; Jiang, Q.; Han, Y. Two amino acid changes in the R3 repeat cause functional divergence of two clustered MYB10 genes in peach. Plant Mol. Biol. 2018, 98, 169-183. [CrossRef]

30. Wang, H.; Zhang, H.; Yang, Y.; Li, M.; Zhang, Y.; Liu, J.; Dong, J.; Li, J.; Butelli, E.; Xue, Z.; et al. The control of red colour by a family of MYB transcription factors in octoploid strawberry (Fragaria $\times$ ananassa) fruits. Plant Biotechnol. J. 2019, 18, 1169-1184. [CrossRef]

31. Härtl, K.; Denton, A.; Franz-Oberdorf, K.; Hoffmann, T.; Spornraft, M.; Usadel, B.; Schwab, W. Early metabolic and transcriptional variations in fruit of natural white-fruited Fragaria vesca genotypes. Sci. Rep. 2017, 7, srep45113. [CrossRef] [PubMed]

32. Symons, G.M.; Chua, Y.-J.; Ross, J.J.; Quittenden, L.; Davies, N.; Reid, J.B. Hormonal changes during non-climacteric ripening in strawberry. J. Exp. Bot. 2012, 63, 4741-4750. [CrossRef]

33. Aharoni, A.; O'Connell, A.P. Gene expression analysis of strawberry achene and receptacle maturation using DNA microarrays. J. Exp. Bot. 2002, 53, 2073-2087. [CrossRef] [PubMed]

34. Sánchez-Sevilla, J.F.; Vallarino, J.G.; Osorio, S.; Bombarely, A.; Posé, D.; Merchante, C.; Botella, M.Á.; Amaya, I.; Valpuesta, V. Gene expression atlas of fruit ripening and transcriptome assembly from RNA-seq data in octoploid strawberry (Fragaria $\times$ ananassa). Sci. Rep. 2017, 7, 13737. [CrossRef] [PubMed]

35. Hong, J.P.; Suh, H.Y.; Kim, J.H.; Ahn, Y.K.; Kim, J.; Lee, E.S.; Kim, D.S.; Lee, H.E. Expression analysis of five Ara-bidopsis PDLP5 homologous in watermelon subjected to biotic and abiotic stresses. Hortic. Environ. Biotechnol. 2017, 58, 367-375. [CrossRef]

36. Wang, S.; Basten, C.J.; Zeng, Z.B. Windows QTL Cartographer 2.5; Dep. of Statistics, North Carolina State University: Raleigh, NC, USA, 2012. 